\title{
Knowledge and Utilization of Red Flags by Physiotherapists in the Assessment and Management of Patients with Low Back Pain
}

\author{
Bello, B., Ibosiola, B.D. \\ Department of Physiotherapy, Faculty of Medicine, Bayero University Kano \\ Correspondence \\ Bello, B. Department of Physiotherapy, Faculty of Medicine, Bayero University Kano•nurubash@yahoo.com
}

\section{SUMMAR Y}

Proper diagnosis and effective treatment of low back pain (LBP) by physiotherapists are believed to be enhanced by knowledge and utilization of red flags.

This cross-sectional study assessed knowledge and utilization of red flags by physiotherapists in the management of patients with LBP. A total of 50 physiotherapists in Kano State were involved in the study. A modified version of a questionnaire from the Royal College of General Practitioners (1999) was used as a tool for data collection. Data retrieved were analysed using descriptive statistics of frequency and percentage. The majority of the physiotherapists $44(88 \%)$ had knowledge of red flags but only $14(28 \%)$ were putting it to good use. A few red flags, such as age of the patient $9(18 \%)$, and history of trauma $8(12 \%)$, were more often documented, while medical history of cancer, HIV status, as well as history of fever were the least documented and by only $1(2 \%)$ physiotherapist.

It was concluded that physiotherapists have adequate knowledge but poor utilization of red flags in their clinical practice.

KEYWORDS: red flags, physiotherapist, low back pain

\section{INTRODUCTION}

Red flags are recognized as indicators of possible serious spinal pathology and their use is indicated by numerous guidelines for proper assessment and management of low back pain (Ferguson et al, 2010; Greenhalgh and Selfe, 2006; Airaksinen et al, 2004). The Guide to Physical Therapist Practice states that initial patient examination is a comprehensive screening and specific testing process leading to diagnostic classification or, as appropriate, to a referral to another practitioner. Medical screening, associated with the examination and evaluation processes leading to patient referral by physiotherapists to practitioners such as physicians (e.g., medical and osteopathic doctors), is of particular importance when treating patients with complaints of low back pain (LBP) as a serious medical disease may present as a musculoskeletal complaint (APTA, 2001). Considering that patients with LBP constitute a significant proportion of the outpatient population serviced by physiotherapists (Jette et al., 1994; Boissonnault and Di Fabio, 1996; Boissonnault, 1999), vigilance for red flags associated with LBP is very pertinent.

A study by Overmeer et al (2005) reported that $40 \%$ of physicians and $25 \%$ of physiotherapists are unfamiliar with red flags. Two studies of doctors' practices showed low level of documentation of use of red flags (Biossonnault and Koopmeiners, 1994; Dilorio et al, 2000). Similarly, one study of a physiotherapist practice also showed a low level 
of utilization of red flags by physiotherapists (Ramsey et al, 1998). Pamela et al (2007) carried out a study in the USA in which they identified bladder dysfunction, history of cancer, immune suppression, history of trauma, saddle anaesthesia, and lower extremity neurological deficit as the most frequently documented red flags.

There is an increasing role for physiotherapists to recognize serious pathology of the spine so as to achieve meaningful diagnosis and effective management (McKenzie and May, 2003). Knowledge and good use of red flags will be most useful in this area. Studies on the level of knowledge and extent of utilization of red flags in the management of patients with LBP in Nigeria are not readily available. This study therefore investigated knowledge and utilization of red flags in the management of patients with LBP among physiotherapists in Kano State, Nigeria.

\section{MATERIALS AND METHODS}

\section{Study Design}

This study was a cross-sectional survey. The study population included all practicing physiotherapists (with at least 2 years experience of treating LBP) in all government and private established hospitals in Kano with a physiotherapy department. These included: Aminu Kano Teaching Hospital (AKTH), National Orthopaedic Hospital, Dala (NOHD), Murtala Muhammad Specialist Hospital (MMSH), Abdullahi Wase Specialist Hospital, Nasarawa (AWSH), Sir Sunusi Specialist Hospital, Yankaba (SSH), Sauki Physiotherapy Clinic and Excellence Physiotherapy Clinic, Kano.

\section{Setting and Recruitment}

The various hospitals listed above served as the venues for the study. Prior to the study, participants were given the opportunity to ask questions about the study. Participating physiotherapists were assured of confidentiality regarding the results.

\section{Participants}

All 75 practicing physiotherapists in the aforementioned hospitals or clinics in Kano State were given the questionnaire except for five who were unavoidably absent at the time of the study. The questionnaires were filled and returned. No sampling technique was involved, as the total number of physiotherapists was obtained from the database of the Nigeria Society of Physiotherapy, Kano State Chapter and all the physiotherapists were accessible. Physiotherapists were recruited if they treat LBP often and had a minimum of two years experience in the management of LBP.

\section{Procedure}

Ethical approval for the study was sought from the ethical committee of AKTH and permission was granted by the Hospital Management Board to administer the questionnaire in the following hospitals, Murtala Muhammad Specialist Hospital (MMSH), Abdullahi Wase Specialist Hospital, Nasarawa (AWSH), Sir Sunusi Specialist Hospital, Yankaba (SSH), Sauki Physiotherapy Clinic, and Excellence Physiotherapy Clinic, Kano.

\section{Data Collection}

A modified version of a questionnaire from the Royal College of General Practitioners was used as the tool for data collection. The questionnaire was distributed to the participating therapists and later retrieved for analysis.

\section{Data Analysis}

Data were obtained on sex, years of practicing physiotherapy, highest qualification, rank/cadre, as well as knowledge, utilization and documentation of red flags. Data were analysed using descriptive statistics of frequencies and percentages.

For knowledge of red flags, respondents were graded using a modified scoring system as follows:

$$
\begin{aligned}
& \text { 23-38 points - poor knowledge } \\
& \text { 39-76 points - fairly adequate knowledge } \\
& \text { 77-115 points - adequate knowledge }
\end{aligned}
$$

For utilization of red flags respondents were graded based on the following:

$$
\begin{aligned}
& \text { 7-11 points } \quad-\text { poor use of red flags } \\
& \text { 12-21 points } \quad-\text { good use of red flags }
\end{aligned}
$$

The Statistical Package for Social Sciences (SPSS) version 15.0 was used for data analysis.

\section{RESULTS}

A total of 75 questionnaires were distributed in the course of the study, out of which 50 were retrieved. Five (5) 
Knowledge and Utilization of Red Flags by Physiotherapists in Assessment and Management of Low Back Pain

physiotherapists were unavoidably absent while 20 did not meet the inclusion criteria of having at least 2 years of practicing physiotherapy or managing LBP.

Table1. Demographic characteristics of respondents

\begin{tabular}{|c|c|c|}
\hline Characteristic & $\mathrm{n}$ & $\%$ \\
\hline \multicolumn{3}{|l|}{ Gender } \\
\hline Male & 24 & 48 \\
\hline Female & 26 & 52 \\
\hline \multicolumn{3}{|l|}{ Qualification } \\
\hline B.Sc. & 38 & 76 \\
\hline M.Sc. & 12 & 24 \\
\hline $\mathrm{PhD}$ & 0 & 0 \\
\hline \multicolumn{3}{|l|}{ Cadre } \\
\hline PT I & 12 & 24 \\
\hline Snr. PT & 15 & 30 \\
\hline Principal PT & 10 & 20 \\
\hline Chief PT & 10 & 20 \\
\hline AD PT & 3 & 6 \\
\hline Director & 0 & 0 \\
\hline \multicolumn{3}{|l|}{ Years of Experience } \\
\hline $0-4$ & 14 & 28 \\
\hline $5-9$ & 12 & 24 \\
\hline $10-14$ & 13 & 26 \\
\hline $15-19$ & 5 & 10 \\
\hline $20-24$ & 3 & 6 \\
\hline $25-29$ & 3 & 6 \\
\hline
\end{tabular}

Of the fifty participants, 26 were female and 24 male. Thirty-eight had B.Sc/B.PT degrees, while 12 had Masters degrees. None had a $\mathrm{PhD}$ degree in physiotherapy. Twentytwo of the physiotherapists had less than five years experience, 20 had been practicing for between 5-15 years, five had 15- 20 years working experience, while only 3 had more than 20 years experience. The results also show that 19 were in Physiotherapist I position, 13 were Senior PTs, 5 were Chief PTs and 3 were assistant directors of physiotherapy.

Table 2 gives a summary of knowledge and use of red flags among the physiotherapists. The majority of the physiotherapists $(88 \%)$ had adequate knowledge about red flags, but only some were putting it to use. This further buttresses the fact that not everything that is known to be right is actually implemented. When subjected to test (Spearman's correlation), there was a strong positive relationship between knowledge and use of red flags (Spearman's rho $=0.230$ ), and it was not statistically significant $(\mathrm{p}>0.05)$.
Table 2. Knowledge and utilization of red flags

\begin{tabular}{lrll}
\hline Knowledge & $\mathrm{n}(\%)$ & Use & $\mathrm{n}(\%)$ \\
\hline Poor & $5(10)$ & Poor & $36(72)$ \\
Good & $1(2)$ & Good & $14(28)$ \\
Adequate & $44(88)$ & & \\
Total & $50(100)$ & & $50(100)$ \\
\hline
\end{tabular}

$\mathrm{n}=$ frequency; $\%$ = percent of population

Table 3 shows that quite an appreciable number of physiotherapists $(40 \%)$ do not know what to do with patients presenting with red flags, $24 \%$ had other responses which included the use of manual therapy, TENS, rest, etc. A few (2\%) documented the use of heat therapy; all these indicating their poor use of red flags in assessing and managing LBP.

Table 3. Action of physiotherapists towards patients presenting with red flags

\begin{tabular}{lc}
\hline Action of PTs towards pt presenting with red flags & $\mathrm{n}(\%)$ \\
\hline No response & $20(40)$ \\
Use of heat therapy & $1(2)$ \\
Use of exercise therapy & $3(6)$ \\
Referral to physician & $14(28)$ \\
Other responses & $12(24)$ \\
Total & $50(100)$ \\
\hline
\end{tabular}

$\mathrm{n}=$ frequency, $\%=$ percentage of population, $\mathrm{pt}=$ patients

Table 4. Most frequently documented red flags by physiotherapists

\begin{tabular}{lc}
\hline Red flag frequently documented & $\mathrm{n}(\%)$ \\
\hline Age of onset $<20$ or $>50$ years & $9(18)$ \\
Recent history of violent trauma & $8(16)$ \\
Constant progressive, non mechanical pain & $4(8)$ \\
Thoracic pain & $2(4)$ \\
Past medical history of malignant tumour & $7(14)$ \\
Prolonged use of corticosteroids & $2(4)$ \\
Drug abuse, immunosuppresion, HIV & $1(2)$ \\
Systematically unwell & $3(6)$ \\
Unexplained weight loss & $4(8)$ \\
Widespread neurological symptoms & $4(8)$ \\
Structural deformity & $5(10)$ \\
Fever & $1(2)$ \\
Total & $50(100)$ \\
\hline
\end{tabular}

$\mathrm{n}=$ frequency, $\%=$ percent of population 
From the table 4 it can be deduced that physiotherapists documented more on recent history of trauma, past medical history of malignant tumour, and age of onset. Very few physiotherapists took note of fever, weight loss, HIV, saddle anaesthesia during assessment of patients with LBP.

\section{DISCUSSION}

The main objective of this study was to determine the level of knowledge and utilization of red flags by physiotherapists in the assessment of patients with LBP. The outcome of the study revealed that most physiotherapists had adequate knowledge but poor utilization of red flags. This finding was further supported by the responses to the question on "what to do when confronted with a patient presenting with red flags?" Answers to this particular question were evidently unsatisfactory and sometimes disturbing. For example, the majority had no response $(40 \%)$, some $(2 \%)$ suggested the use of heat therapy, which is clearly contraindicated in these patients with red flags, and only a few documented referral to a physician (24\%). Referral to a physician or to the most appropriate medical practitioner has been recognized as the best decision when faced with red flags (Biossonnault and Koopmeiners, 1994; Atlas and Deyo, 2001). This poor utilization of red flags is particularly disturbing because complications that could arise from this inappropriate decision could further worsen the patients' condition and at worst could even result in the patient losing his/her life.

In the past, the profession of physiotherapy relied on the medical profession for recognition of musculoskeletal conditions (Dilorio et al, 2000). Physiotherapists often took the role of technicians carrying out treatments that were prescribed by doctors. Presently, this model is changing. Physiotherapists are increasingly recognized by doctors as experts, particularly in relation to the management of musculoskeletal conditions (Dilorio et al., 2000). The present study showed that adequate knowledge of red flags by physiotherapists must be responsible for this change in the role of physiotherapists in the diagnosis and management of musculoskeletal conditions.

The results of this study also show that physiotherapists who are new in the field were more knowledgeable about red flags than those that had been in the profession much longer. This may be attributed to the improvement and changes currently being seen in the training of physiotherapists. For example, it is obvious that in Nigeria, physiotherapy training has metamorphosed from a four-year B.Sc. Physiotherapy training to a five-year professional Bachelor of Physiotherapy degree. This has undoubtedly had an impact on the clinical and knowledge base of the newly-trained physiotherapists over the earlier practicing physiotherapists who might have gone through a diploma and B.Sc. curriculum that did not lay emphasis on diagnostic reasoning by physiotherapists. Rather they were trained to just treat a patient based on a doctor's prescription or request. The issue of poor continuing physiotherapy education programme also puts most of the earlier practicing physiotherapists, who have not acquired additional qualifications, at a disadvantage as regards gaining new skills and information to update their knowledge base.

Specific red flags were also observed to be more documented than others; red flags relating to recent history of violent trauma and past medical history of malignant tumour, and age of the patient, were most commonly documented. This is similar to the results of a previous study by Ramsey et al (1998) in which red flags frequently documented included: age of the patient, saddle anaesthesia, history of trauma, medical history of cancer, and presence of night pain. The least documented red flags were fever, weight loss, HIV, and drug abuse. This might be due to poor attention on these forms of red flags and the wrong conception that LBP patients do not ordinarily report such cases to physiotherapists. Similarly, there could be possible reluctance on the part of physiotherapists to question certain patients (e.g. the elderly, the very young, those with high social status, religious clerics) about their HIV status and the use of drugs.

\section{CONCLUSION AND RECOMMENDATION}

Physiotherapists have adequate knowledge but poor use of red flags in their clinical practice. It is recommended that physiotherapists should engage in the documentation of red flags in the management of patients with LBP and promptly refer patients with red flags to the appropriate specialty.

\section{References}

American Physical Therapy Association. Guide to Physical Therapist Practice. 2001. 2nd ed. Physical Therapy, pp746.

Atlas, S.J, and Deyo, R.A. 2001. Evaluating and managing acute low back pain in the primary care setting. Journal of General Internal Medicine 16: 120-131. 
Knowledge and Utilization of Red Flags by Physiotherapists in Assessment and Management of Low Back Pain

Airaksinen, O., Brox, J.I., Cedraschi, C. 2004. European guidelines for the management of chronic non-specific low back pain. European Commission, Research Directorate General; Retrieved from http://www.backpaineurope.org on 04/07/2012.

Biossonnault, W.G. and Koopmeiners, M.B. 1994. Medical history profile: Orthopedic physical therapy out patients. Journal of Orthopedic and Sports Physical Therapy 20: 2-10.

Boissonnault, W.G 1999. Prevalence of co-morbidities, surgery, and medication use in a physical therapy outpatient population: A multi-centered study. Journal of Orthopedic and Sports Physical Therapy 29: 506-525.

Boissonnault, W.G and Di Fabio, R.P. 1996. Pain profile of patients with low back pain referred to physical therapy. Journal of Orthopedic and Sports Physical Therapy 24: 180-191.

Dilorio, D., Henley, E. and Doughty, A. 2000. A survey of primary care physician practice patterns and adherence to acute low back problem guidelines. Archives of Family Medicine 9: 1015-1021.

Ferguson, F., Holdsworth, L. and Rafferty, D. 2010. A national framework for supporting improvement in the physiotherapy assessment and management of low back pain: the Scottish experience. Physiotherapy 96: 198-205.
Greenhalgh, S., Selfe, J. 2006. Red Flags: A guide to identifying serious pathology of the spine. Edinburgh: Churchill Livingstone.

Jette, A.M., Smith, K., Haley, S.M., Davis, K.D. 1994. Physical therapy episodes of care for patients with low back pain. Physical Therapy 74: 101-110.

McKenzie, R. and May, S. 2003. Construct validity of lumbar extension measures in McKenzie's derangement syndrome. An International Journal of Musculoskeletal Therapy 12: 328-334.

Overmeer, R., Thomas, M.S., Linton, D. et al. 2005. Do evidence based guidelines have an impact in primary health? A cross sectional study of Swedish physician and physiotherapist. Health Service Research Journal 30: 146-151.

Pamela, J., Leerar, P.T., Biossonnault, W., Domholdt, E. and Roddey, T. 2007. Documentation of red flags by physical therapist for patient with low back pain. Journal of Manual and Manipulative Therapy 15: 42-49.

Ramsey, P.G., Curtis, R., Pauuw, D. S., Carline, J.D. and Wenrich, M.D. 1998. History taking and preventive medicine skills amongst primary physicians: An assessment using standardized patient. American Journal of Medicine 104: 152158. 